C'est sur le nitrate de chaux de cette provenance, à $13 \%$ d'azote, que ¡iai expérimenté. L'essai a porté en même temps sur des nitrites. La fabrication visée plus haut donne une partie d'azote oxydé à l'état de nitrite de chaux, qu'elle transforme en nitrate, les nitrites ne se consommant pas actuellement comme engrais. Mais faut-il réellement proscrire les nitrites? Sont-ils nuisibles à la végétation, sonttls utilisés par elle? Voici quelques résultats d'expériences sur ces questions :

Culture, en pots, de mais jaune gros (juillet-octobre I905).

(Dans chaque lot, 9 kilogr. terre de Boulogne + ogr $405 \mathrm{P}^{2} \mathrm{O}^{5}$ (superphosphate) + I 500 sulfate de potasse. Nitrates et nitrites ajoutés, en dehors des témoins, à raison de ogr 200 d'azote pour 9 kilogr. de terre, soit de 80 kilogr. environ à l'hectare).

Poids des récolles sicches

Témoins sans addition d'azote ............ on grammes

Nitrite de soude et nitrite de chaux (moyenne). 54,6

Nitrate de soude.................... 54,2

Nitrate de chaux de Norvège............. 54,0

Sous la même dose d'azote, nitrates et nitrites mis en ceuvre se sont montrés également efficaces; la perfection de l'égalité est peutêtre, d'ailleurs, un peu fortuite. Ces expériences sont à continuer.

\section{Appareillage à haute tension}

Dans les installations à haute tension, un choix judicieux de l'appareillage est d'une importance primordiale. Aussi nous proposonsnous aujourd'hui de donner à nos lecteurs quelques renseignements généraux sur divers types d'appareils d'un emploi courantqui sont construit par la Société Indus trielle des Téléphones.

Les interrupteurs pour la haute tension peuvent se diviser en trois catégories principales : les appareils à grande distance de rupture dans l'air, les appareils à rupture sur corne, et les appareils à rupture dans l'huile.

La première catégorie d'appareils comprend les types dits à couteaux cintrés, appareils très simples convenant à des tensions inférieures à 6.000 volts et à des puissances ne dépassant pas $250 \mathrm{ki}-$ lowatts. Ils comprennent ún dispositif de commande par levier avec un palier en bronze poli et une poignée d'ébonite, et sont montés sur des socles en marbre blanc munis de douilles en porcelaine. Des cloisons en ardoise huilée sont disposées entre chaque pôle; les couteaux sont en bronze, accouplés par une barre de gaïac à double isolement de mica; les mâchoires sont en cuivre rouge et aemontables. Enfin ces appareils peuvent être munis de pareétincelles en charbon.
Dans la même catégorie, mais pour des tensions plus éle. vées (jusqu'à 1.000 kilowatts et 10.000 volts), la Société Industrielle des Téléphones construit des types à leviersquí se distinguent surtout des précédents parce que les couteaux de bronze sont remplacés par des leviers en frêne verni portant à l'intérieur un câble souple, et montés à ser: rage dans des pièces de bronze fixées sur un axe carré avec interposition d'un fort isolement de mica et de prespahn.

Les appareils à ruptures sur corne peuvent s'employer jusqu'à $\mathrm{I} 0.000$ volts; ils comportent une commande par levier avec un palier en bronze poli et une poignée d'ébo. nite. Des couteaux pivotants en cuivre rouge, montés sur, des porcelaines, viennent s'engager dans des mâchoires en cuivre rouge également montées sur des porcelaines et fáci. lement démontables. (Voir figure I).

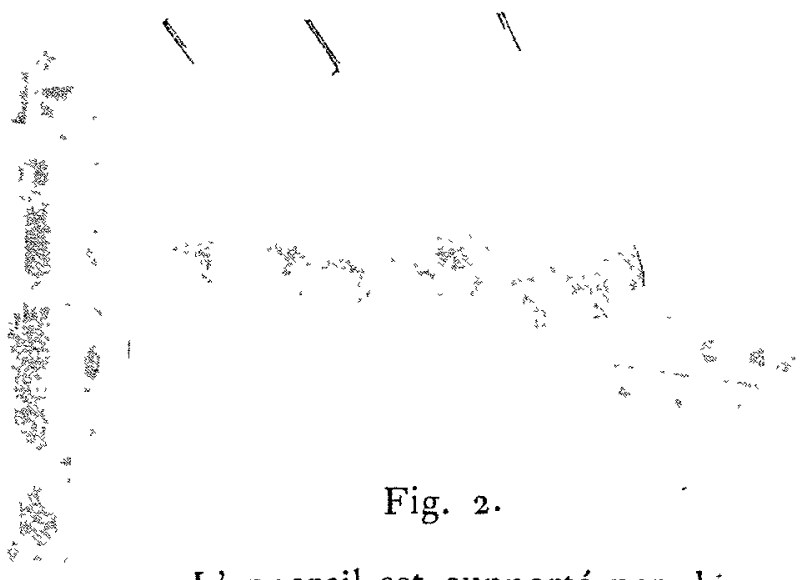

L'appareil est supporté par dés consoles en fonte de fer disposées pour permettre un montage facile derrière les panneaux des tableaux de distribution.

$\mathrm{Ce}$ modèle subit de légères modifications pour les installations à l'extérieur.

Dans ce cas, les pôles sont montés sur deux cornières entretoisées par deux pièces en fonte qui servent de paliess: à l'axe des couteaux mobiles. Ces cornières sont fixées aux poteaux par des boulons et des ferrures qui constituent des jambes de force et assurent la rigidité de l'ensemble. Les interrupteurs de ce type peuvent être montés: soit en porteà-faux, sur un seul poteau, soit entre deux poteaux, soit enfin. au sommet d'un poteau unique. Dans ce dernier cas, un chapeau en fonte avec boulons embrasse l'extrémité supé rieure du poreau et porte les bras sur lesquels sont boulonnées les cornières de l'appareil. La commande peut se faire par chaîne, par tringle ou par une manivelle de sûreté amo. vible. (Voir figure 2).

Enfin les interrupteurs de troisième catégorie, à rupture dans thuile, se divisent en deux séries. La première com. prend les appareils destinés aux puissances inférieures 25 o kilowatts, sous une tension de 6.000 volts au maximum. Ils sont à bac unique, la commande est rotative et comporte un dispositif de genouillères et de ressorts de rappel; l'iso. lement des couteaux et mâchoires 'est particulièrement șolgné et les distances de rupture sont largement calculées; les prises de courant sont protégées par des porcelaines de forme spéciale; enfin l'appareil peut être disposé pour être fixé, au gré des intéressés, soit sur un tableau, soit contre un mur.

Les interrupteurs dans l'huile de la deuxième série comt portent autant de bacs que de pôles et ces bacs sont revêtus. intéricurement de garnitures isolantes. La rupture se fait en deux points sur chaque pôle. L'entrée et la sortie sont? 
protégées par des porcelaines de grande dimension, dont l'isolement est éprouvé au cours de la construction. Ces porcelaines renferment èlles-mêmes des tubes de micanite concentriques qui assurent un isolement absolument parfait. Les pièces de contact, supportées elles-mêmes par des porcelaines, sont scellées au plomb et par suite ne peuvent être descellées par l'huile chaude. Le système de commande est rotatif avec des genouillères et des ressorts de rappel puissants, de telle sorte que l'interrupteur ne puisse rester dans une position intermédiaire entre la position d'ouverture et celle de fermeture.

Enfin, le montage sur les tableaux de distribution est particulièrement facile.

La Société Industrielle des Téléphones a exécuté des appareils de cette série pour des tensions atteignant 30 ooo volts et des puissances de 2 ooo kilvoltampères.

Les interrupteurs dans l'huile qui viennent d'être décrits se prêtent particulièrement bien à l'adjonction de dispositifs de déclenchement, avec ou sans retardateurs.

On sait en effet que l'emploi des disjoncteurs à maximum se répand de jour en jour davantage et que, dans les grandes stations centrales, ces appareils tendent à remplacer complètement les coupe-circuits dont les inconvénients sont bien connus.

Le déclenchement est produit par une ou plusieurs bobines branchées sur des transformateurs d'intensite; le noyau de ces bobines agit directement, par choc, sur les organes de déclenchement. Ce dispositif présente l'avantage de n'exiger ni source de courant spéciale, ni isolement particulier, le courant qui passe dans les bobines étant à faible tension.

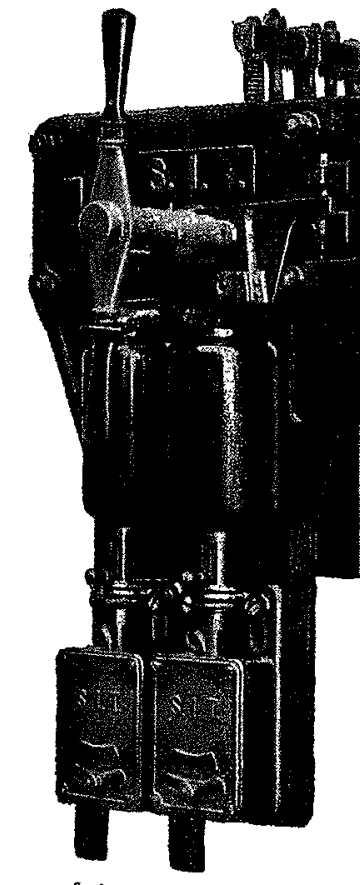

Lorsqu'on veut éviter le fonctionnement intempestif des disjoncteurs, par suite d'un à coup momentané ou d'un court circuit de durée insignifiante, on munit les disjoncteurs qui viennent d'être décrits, de retardateurs réglables. Ceux-ci se composent essentiellement d'un dash-pot à huile, dont le mouvement est transmis, par un système de bielles et de leviers démultiplicateurs, à un crochet qui retient le noyau Fig. 3. de la bobine de déclenchement et ne le lâche qu'au bout d'un certain temps à peu près indépendant de l'intensité de la surcharge. En déplaçant le point d'oscillation du levier porte-crochet ce qui se fait au moyen d'un bouton qui glisse dans une fente étalonnée, on peut faire varier la durée de l'accrochage, c'est-à-dire le retard, de o à i 5 secondes. Il est inutile d'insister sur les avantages de cette faculté de réglage. Elle permet notamment de régler dans une installation comportant plusieurs feeders, de régler les disjoncteurs qui commandent ces feeders, pour un retard inférieur à celui du disjoncteur principal ; on évite ainsi qu'un accident affectant un seul point du réseau n'immobilise toute l'installation. Le fonctionnement de ces retarfateurs est purement mécanique, ce qui évite l'emploi d'une source d'énergie spéciale; ils sont en outre robıste et facile à régler.
Les coupe-circuits les plus courants sont à poignée de porcelaine; ils sont d'une construction simple et robuste; la partie fixe comprend deux mâchoires montées sur des porcelaines spéciales qui sont elles-mêmes fixées sur une ferrure inclinée, empêchant toute possibilité d'ouverture accidentelle sous l'influence de vibrations. La partie mobile qui contient le fusible est munie de couteaux destinés à pénétrer dans les mâchoires de la partie fixe; elle peut pivoter autour d'un axe dans la chape inférieure, ce qui facilite la mancuvre de mise en place; le fusible d'argent, de cuivre ou d'aluminium, est placé dans un tube d'amiante qui empêche toute rupture de pnrcelaine au moment de la fusion.

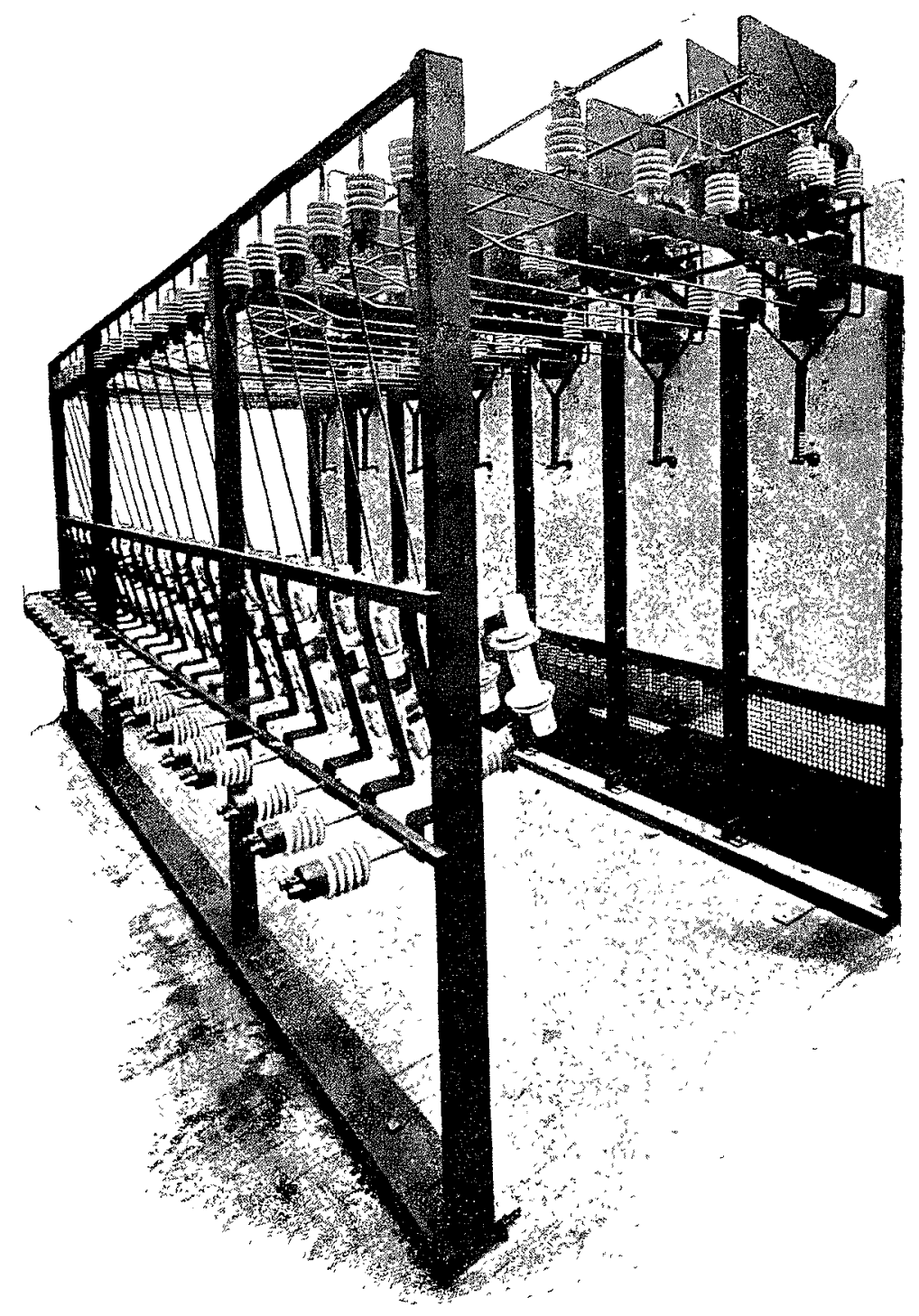

Fig. 4.- Appareillage d'un tableau de distribulion

Dans la construction des tableaux de distribution qui lui ont été confiés, la Société des Téléphones s'est appliquée à rendre facile l'accès et la visite des connexions; par un groupement judicieux des appareils elle a pu réaliser un schéma de connexions simple et commode et présentant toutes les garanties de sécurité désirable: la vue ci-contre montre les connexions d'un tableau destiné à une distribution triphasée à 5 ooo volts

On remarquera que l'on peut, sans aucun danger, circuler derrière le tableau et que l'accès des coupe-circuits, pour le remplacement éventuel des fusibles, est particulièrement facile.

M. P. 